\section{Hier steht eine Anzeige.}

Springer

\title{
Zwangsaufkauf \\ Praxisnachfolge: Kleine Praxen geraten unter Druck
}

\section{Chefs kleiner Praxen müssen sich besonders gut wappnen, wollen sie einem Zwangsaufkauf durch die Kassenvereinigung zuvorkommen.} Klappt es nicht, ist auch die Entschädigung zumindest steuerlich zu prüfen.

$D$ ie Inhaber von sehr kleinen Praxen in überversorgten Gebieten könnten durch das GKV-Versorgungsstärkungsgesetz ein Problem bekommen, ihre Praxis an einen Nachfolger abzugeben. Deshalb sollten sie rechtzeitig Vorkehrungen treffen, rät der Medizinrechtler Dirk Niggehoff von der Kanzlei Möller und Partner. „In einem solchen Fall sollte man dringend überlegen, wie man die Braut hübscher machen kann", sagte Niggehoff bei einer Informationsveranstaltung der Steuerberater- und Wirtschaftsprüferkanzlei Laufenberg Michels und Partner in Köln.

\section{Fallzahl ist entscheidend}

Handlungsbedarf könnte entstehen, wenn der Versorgungsgrad $140 \%$ überschreitet und die Praxis bei den Fallzahlen deutlich unter dem Fachgruppendurchschnitt liegt. Dann könnte der Zulassungsausschuss die Nachbesetzung ablehnen, wenn keine besonderen Versorgungsaspekte für eine Fortführung der Praxis sprechen. Um zu wissen, ob das für ihre Praxis zutreffen könnte, sollten niedergelassene Ärzte rechtzeitig ihre KV fragen, empfahl Niggehoff.

Wenn Ärzte einen Kollegen anstellen oder einen Partner aufnehmen wollen, um diesem die Praxis zu verkaufen, sollten sie das rechtzeitig tun. Denn der Zulassungsausschuss muss nur dann auf jeden Fall einem Nachbesetzungsverfahren zustimmen, wenn die Konstellation bereits seit mindestens drei Jahren besteht. Gerade bei sehr kleinen Praxen sei es aber oft fraglich, ob sich das überhaupt lohnt, so der Jurist. Für die betroffenen Ärzte werde es deshalb in Zukunft wichtiger, den Versorgungsauftrag der Praxis auszufüllen, sagte Oliver Pellarin, Niederlassungsberater der KV Nordrhein (KVNo). Sie sollten der KV jede längere Abwesenheit aus
Krankheit oder sonstigen Gründen melden.

Grundsätzlich sei es bei einem Versorgungsgrad von über $140 \%$ notwendig, Zusatzargumente für den Verbleib der Praxis am Markt zu finden. Das könne etwa ein besonderes Versorgungsangebot sein oder die Tatsache, dass die spezielle Fachrichtung in einem MVZ benötigt wird. Wenn Ärzte während der Sitzung des Zulassungsausschusses erkennen, dass die Nachbesetzung wohl abgelehnt wird, sollten sie ihren Antrag zurückziehen, riet der KVNo-Experte.

Grundsätzlich sei es im Sinne der KV, gemeinsam mit den Ärzten eine Lösung zu finden - nicht zuletzt, da sie sonst eine Entschädigung zahlen muss. „Welches Interesse sollte die KV haben, Geld aus der Gesamtvergütung zu nehmen, um damit Praxen aufzukaufen?" fragte Pellarin.

\section{Der Fiskus verdient mit}

Erhält ein Arzt, dessen Praxis nicht nachbesetzt wird, von der KV eine Entschädigung, so ist diese einkommensteuerpflichtig, erläuterte der auf Heilberufe spezialisierte Steuerberater Dr. Rolf Michels. Unter bestimmten Voraussetzungen werde aber nur der halbe Steuersatz fällig. Das sei dann der Fall, wenn der Arzt älter als 55 Jahre oder dauernd berufsunfähig ist. Zudem muss er die freiberufliche Tätigkeit zunächst beenden.

Die von der KV gezahlte Entschädigung ist nach Ansicht von Michels auch nicht umsatzsteuerpflichtig. Anders könne das allerdings bei einer „Teilentschädigung “ aussehen, wenn der Praxisinhaber etwa den privatärztlichen Teil an einen Kollegen veräußert. „Das muss auf jeden Fall ein Steuerberater prüfen“, sagte er.

Ilse Schliengensiepen 